\title{
Sublingual epidermoid cyst: unusual diagnosis in Otorhinolaringology
}

\begin{abstract}
Dermoid and epidermoid cysts are rare in the head and neck, specially in the oral cavity, accounting for $1.6 \%$. It represents an even smaller percentage of all the oral cysts, less than $0.01 \%$. Usually asymptomatic, however, occasionally, obstructive symptoms like dysphagia or speech difficulties are found. We present a case of a 32 years old female patient with a sublingual epidermoid cyst. The patient was referred to our ENT department with complaints of an oral mass, speech difficulties and solid food dysphagia for 1 month. Physical examination revealed a well circumscribed symmetrical mass on the floor of the mouth with no inflammatory signs, tender and fluctuant on palpation. Computed tomography (CT) was requested. CT scan showed a sublingual cystic mass inferiorly limited by the mylohyoid muscle. Aspiration was performed to improve symptoms until surgery. The liquid that resulted from aspiration showed a granulated white cheesy material. Enucleation of the cyst was performed intraorally in the operating room. Histopathologic investigation confirmed the diagnosis of epidermoid cyst. The patient fully recovered and there was no recurrence. Although rare, cases like this exist. There are several differential diagnoses and a high level of suspicion is necessary for a correct workup, diagnosis and follow-up. Surgical excision is usually necessary and curative.
\end{abstract}

Keywords: Epidermoid cyst, Dermoid cyst, sublingual, enucleation
Volume I3 Issue | - 202|

\author{
Hugo Marcos, Tiago Soares Santos, Paulo \\ Gonçalves, Débora Gonçalves, Guilherme \\ Carvalho, Vera Miranda, Carlos Carvalho \\ ENT Department of Hospital de São Sebastião, Portugal
}

Correspondence: Guilherme Carvalho, ENT Department of São Sebastião Hospital, R. Dr. Cândido Pinho, 4520-2II Santa Maria da Feira, Portugal, Tel +35 I256377900, Fax +35I 256373876, ORCID 0000-000 I-6282-9795, Email guilherme.machado.carvalho@lusiadas.pt

Received: November 02, 2019 | Published: February 03, 202

\section{Introduction}

Dermoid and epidermoid cysts are benign lesions found in various body locations, with an estimated frequency of $7 \%$ in the head and neck and $1.6 \%$ in the oral cavity. ${ }^{1,2}$ When located on the floor of the mouth, they are believed to be most commonly the result of a defect in embryogenesis regarding the fusion in the midline of the first and second branchial arches. ${ }^{2,3}$ However, two possible etiopathogeneses are recognized: acquired implantation and congenital inclusion. ${ }^{4}$ Histologically, they can be divided into three types: epidermoids, dermoids and teratoids ${ }^{5,6}$ with epidermoid cysts being the most common and teratoids being the least common. ${ }^{7}$ Epidermoid cysts are composed of a stratified squamous epithelium membrane without dermal components; dermoid cysts are composed of a stratified squamous epithelium membrane with dermal components inside including sebaceous glands, sweat glands and hair follicles; and teratoid cysts are composed of a stratified squamous epithelium membrane with elements from the three germ layers (ectoderm, mesoderm and endoderm) inside. ${ }^{6}$

In terms of demographic distribution, there is no gender predilection and they are usually diagnosed in the second and third decades of life, although there are cases described from 7 months to 77 years of age. ${ }^{4,8}$ Its growth is usually slow and progressive. The symptoms are related to the swelling volume. Treatment is surgical and a transoral or transcervical approach can be used, according to the location and size of the lesion. ${ }^{8}$ In this article, the authors present the case of a 32-year-old female patient with an asymptomatic sublingual epidermoid cyst undergoing surgical treatment.

\section{Case presentation}

A female patient, 32 years old, non-smoker, went to the emergency department of our Otorhinolaryngology department with complaints of oral swelling, speech difficulties and progressive dysphagia for solids with about a month of evolution. No pain complaints, no dyspnea, no fever or similar episodes before. The patient also denied any surgical history or trauma to the oral cavity and any other relevant medical or personal history. On objective examination, a well-defined, symmetrical sublingual swelling was found, with no inflammatory signs, covered by normal-looking mucosa and about $3 \mathrm{~cm}$ in diameter (Image 1). Upon palpation, the lesion showed a soft consistency, with fluctuation and no associated pain. There were no signs of ulceration or other associated injuries. Due to the volume of the lesion, the tongue was displaced posteriorly. Upon cervical palpation, no adenopathies or other changes were identified. A CT scan was requested to better characterize the lesion in which a cystic mass about $6 \mathrm{~cm}$ in diameter could be seen, with homogeneous content located in the sublingual region, inferiorly limited by the mylohyoid muscle, with no relief after contrast (Image 2). No evidence of calculations or dilation of the submandibular duct, neither invasion of adjacent structures or adenopathies were found.
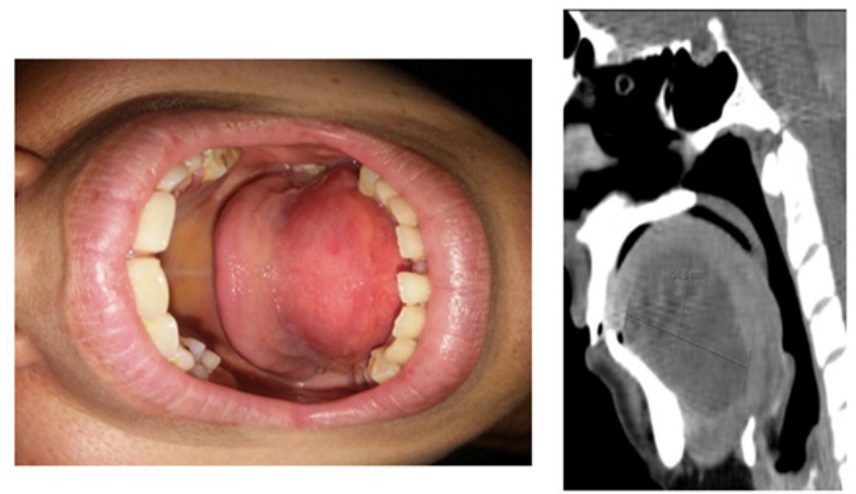

In addition, an aspiration puncture was performed not only for symptomatic relief but also for the histological characterization of the lesion. The aspirated content had a granular, whitish and viscous appearance that was difficult to aspirate (Image 3). 


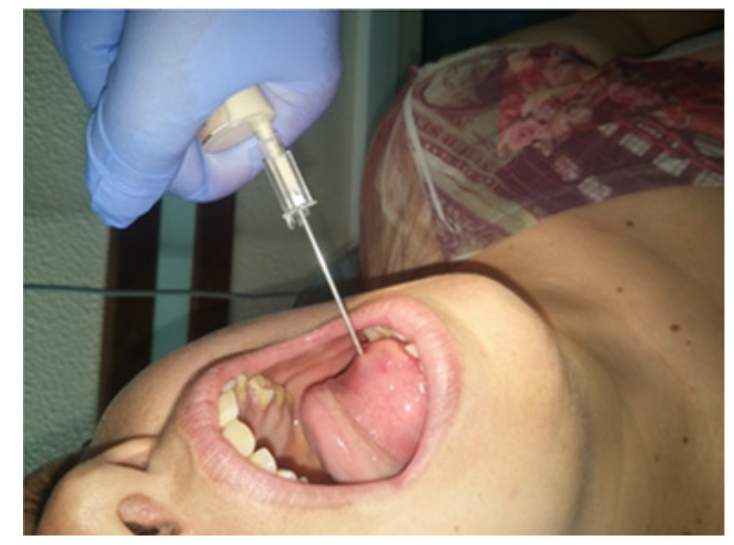

Under general anesthesia and nasotracheal intubation, the patient underwent transoral enucleation of the cyst. The cyst was dissected from the surrounding muscular tissue and mucosa, preserving the integrity of the capsule (Image 4).
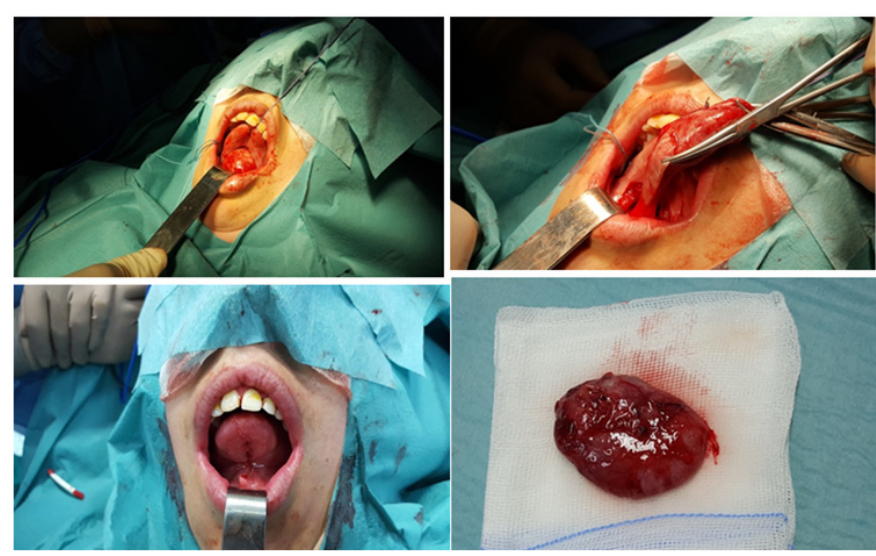

The surgery was uneventful and the tongue returned to its normal position. The histological result was an epidermoid cyst. One year after surgery, the patient was asymptomatic and without clinical evidence of recurrence.

\section{Discussion}

Sublingual or floor of the mouth cysts may be congenital or acquired. ${ }^{4}$ In the present case, it is most likely to be a congenital epidermoid cyst due to its location in the midline and the absence of a previous history of surgery or trauma. Due to its slow and progressive growth, the symptoms often appear after several years of existence. Not rarely, the patient only perceives his existence when he already has a considerable volume. So, as we could see previously, the patient has been reporting symptoms for about a month, despite the swelling already being about $6 \mathrm{~cm}$, without inflammatory/infectious signs that support a sudden growth. Due to the particularly low frequency of this type of pathology, a high degree of suspicion and a solid knowledge of possible differential diagnoses is mandatory for a correct investigation and, consequently, a correct diagnosis. In our patient, the absence of local inflammatory / infectious signs, coupled with the absence of pain that evolved over a period of at least one month makes the possibility of an infection, such as Ludwig's Angina, very unlikely. On the other hand, the hypothesis of being a malignant tumor was not considered as probable since it is a young patient, with no known risk factors, with a lesion without suspicious clinical characteristics, without invasion of adjacent structures or cervical adenopathies. .
Thus, our main suspicions were ranula or embryonic defect. The fact that the swelling was symmetrical removed some strength from the hypothesis of being a ranula. Table 1 summarizes the main differential diagnoses of oral and / or cervical swelling. ${ }^{9}$

Table I Differential diagnoses of mouth or neck swellings ${ }^{7}$

\begin{tabular}{ll}
\hline Category & Lesion \\
\hline Developmental & Dermoid cyst Ectopic thyroid \\
& Branchial cleft cyst \\
& Ectopic thyroid \\
& Sublingual abscess \\
Sublingual cellulitis & Ludwig's angina \\
Infection & Sublingual sialadenitis \\
& Mucocele \\
& Ranula \\
Salivary gland & Unilateral or bilateral Wharton 's duct blockage \\
& Salivary gland tumour (benign or malignant) \\
& Cystic hygroma \\
& Squamous cell carcinoma \\
& Other benign \& malignant (lipoma, \\
neurofibroma, haemangioma, lymphangioma) & Epidermoid cyst \\
Oral lymphoepithelial cyst \\
Hamartomatous
\end{tabular}

Imaging exams are an important aid in the diagnosis and surgical planning. Ultrasonography, CT scan and magnetic resonance imaging (MRI) are reported in the literature as being useful, with preference given to the last two for the better characterization of the deep limits of the lesion. ${ }^{1}$ Aspiration puncture, although unnecessary, can be important for direct observation of the cyst content, in its histological distinction and for symptomatic relief in cases with large cysts. ${ }^{10}$ Treatment is surgical and the approach will depend mainly on two factors: the dimensions of the cyst and its location. ${ }^{11}$ Cysts up to $6 \mathrm{~cm}$ with supra-mylohyoid location are preferably treated transorally, with evident aesthetic benefits. In turn, cysts with infra-mylohyoid location or extension will most likely need a cervical approach. ${ }^{8}$ The size of the cyst alone does not dictate the approach. ${ }^{12}$ The prognosis after surgical excision is excellent, with very low recurrence rates. ${ }^{4}$

\section{Acknowledgments}

None.

\section{Conflicts of interest}

The author declares that there is no conflict of interest to disclose.

\section{Funding}

None. 


\section{References}

1. Turetschek K, Hospodka H, Steiner E. Case report: epidermoid cyst of the floor of the mouth: diagnostic imaging by sonography, computed tomography and magnetic resonance imaging. $\mathrm{Br} \mathrm{J}$ Radiol. 1995;68(806):205-207.

2. Verma S, Kushwaha JK, Sonkar AA, et al. Giant sublingual epidermoid cyst resembling plunging ranula. Natl J Maxillofac Surg. 2012;3(2):211213.

3. Jham BC, Duraes GV, Jham AC, et al. Epidermoid cyst of the floor of the mouth: a case report. J Can Dent Assoc. 2007;73(6):525-528.

4. Kyriakidou E, Howe T, Veale B, et al. Sublingual dermoid cysts: case report and review of the literature. J Laryngol Otol. 2015;129(10):10361039.

5. Meyer I. Dermoid cysts (dermoids) of the floor of the mouth. Oral Surg Oral Med Oral Pathol. 1955;8(11):1149-1164.

6. Gordon PE, Faquin WC, et al. Floor-of-mouth dermoid cysts: report of 3 variants and a suggested change in terminology. J Oral Maxillofac Surg. 2013;71(6):1034-1041.
7. Yilmaz I, Yilmazer C, Yavuz H, et al. Giant sublingual epidermoid cyst: a report of two cases. J Laryngol Otol. 2006;120(3):E19.

8. Longo F, Maremonti P, Mangone GM, et al. Midline (dermoid) cysts of the floor of the mouth: report of 16 cases and review of surgical techniques. Plast Reconstr Surg. 2003;112(6):1560-1565.

9. MacNeil SD, Moxham JP. Review of floor of mouth dysontogenic cysts. Ann Otol Rhinol Laryngol. 2010;119(3):165-173.

10. Jain H, Singh S, Singh A. Giant sublingual dermoid cyst in floor of the mouth. J Maxillofac Oral Surg. 2012;11(2):235-237.

11. El-Hakim IE, Alyamani A. Alternative surgical approaches for excision of dermoid cyst of the floor of mouth. Int J Oral Maxillofac Surg. 2008;37(5):497-499.

12. Boko E, Amaglo K, Kpemissi E. A bulky dermoid cyst of the floor of the mouth. Eur Ann Otorhinolaryngol Head Neck Dis. 2014;131(2):131134 